\title{
Barrel cortical neurons and astrocytes coordinately respond to an increased whisker stimulus frequency
}

\author{
Jun Zhao ${ }^{1,2+}$, Dangui Wang ${ }^{1,3+}$ and Jin-Hui Wang ${ }^{1,2,3^{*}}$
}

\begin{abstract}
Background: Nerve cells program the brain codes to manage well-organized cognitions and behaviors. It remains unclear how a population of neurons and astrocytes work coordinately to encode their spatial and temporal activity patterns in response to frequency and intensity signals from sensory inputs.

Results: With two-photon imaging and electrophysiology to record cellular functions in the barrel cortex in vivo, we analyzed the activity patterns of neurons and astrocytes in response to whisker stimuli with increasing frequency, an environmental stimulus pattern that rodents experience in the accelerated motion. Compared to the resting state, whisker stimulation caused barrel neurons and astrocytes to be activated more synchronously. An increased stimulus frequency up-regulated the activity strength of neurons and astrocytes as well as coordinated their interaction. The coordination among the barrel neurons and astrocytes was fulfilled by increasing their functional connections.
\end{abstract}

Conclusions: Our study reveals that the nerve cells in the barrel cortex encode frequency messages in whisker tactile inputs through setting their activity coordination.

Keywords: Neuron, Astrocyte, Barrel cortex, Whisker and two-photon cellular imaging

\section{Background}

Brain functions are fulfilled by the activities of nerve cells [13]. The emerging evidences demonstrate that the neurons and astrocytes are involved in the information processing in the central nervous system [4-8]. Their communications are critical for the brain functions, such as the astrocytes are able to regulate the neuronal activities and plasticity [9-12]. However, how these neurons and astrocytes in local networks communicate coordinately to program the brain codes for well-organized cognition and behaviors remains unclear. The address of this issue helps to reveal the principles of information processing in the brain.

The studies by two-photon cellular imaging show that sensory stimuli, such as vision and auditory, activate the groups of neurons and astrocytes in sensory cortices [6,8,13-16]. Their temporal and spatial activity patterns in response to a single stimulation are well

\footnotetext{
* Correspondence: jhw@sun5.ibp.ac.cn

${ }^{\dagger}$ Equal contributors

1 Institute of Biophysics, Chinese Academy of Sciences, Beijing 100101, China

${ }^{2}$ Graduate School of the Chinese Academy of Sciences, Beijing 100049, China

Full list of author information is available at the end of the article
}

known [17-21]. In fact, the sensory inputs include the messages of frequency and intensity [22-25]. How the neurons and astrocytes in cortical networks coordinately work to recognize such messages in sensory inputs remains elucidated.

The nerve cells in the barrel cortex receive whisker tactile inputs [26-28], which is well used to study how the nerve cells in barrel cortical networks encode the whisker inputs. With the approaches of two-photon cellular imaging and electrophysiology in vivo, we examined the temporal and spatial activity patterns of barrel neurons and astrocytes in response to an increasing stimulus frequency to the whiskers, a common external stimulation when rodents explore their environments, such as the accelerated motion [23,25]. By analyzing the magnitudes and temporal correlation of responses among the neurons and astrocytes, we found that neurons and astrocytes in barrel cortex coordinately respond to an increased whisker stimulus frequency.

\section{Biomed Central}

(c) 2012 Zhao et al.; licensee BioMed Central Ltd. This is an Open Access article distributed under the terms of the Creative Commons Attribution License (http://creativecommons.org/licenses/by/2.0), which permits unrestricted use, distribution, and reproduction in any medium, provided the original work is properly cited. 


\section{Results}

Local field potentials were recorded in the barrel cortices of mice to reveal how sensory neurons recognize frequency messages in whisker tactile input. We subsequently studied how the neurons and astrocytes coordinately respond to the frequency messages by analyzing their activity strength and synchrony with twophoton calcium imaging in vivo. The barrel cortices for the field potential recording and two-photon cellular imaging were localized as illustrated in Figure 1A $\sim$ B. The levels of $\mathrm{Ca}^{2+}$ signal in the cells were presumably proportional to their activities [19,29-32]. Sulforhodanmin-101 (SR-101) that labeled the astrocytes [33] was used to isolate astrocytic signal from neuronal one. The frequency messages in whisker tactile inputs were to stimulate mouse whiskers by paired burststimuli with increasing frequency (stimulus one at $8 \mathrm{~Hz}$ and stimulus two at $12 \mathrm{~Hz}$, i.e., 8-to- $12 \mathrm{~Hz}$ ). The spatial patterns of cellular activity were analyzed by the portions of $\mathrm{Ca}^{2+}$ signal patterns, and the synchrony was estimated by pair-wise crosscorrelation.

The activity of barrel neurons rises in response to an increased frequency of whisker tactile input

By recording local field potentials (LFP) in the barrel cortex, we studied the responses of neurons to whisker stimuli in frequencies 8-to-12 Hz. Figure 1C illustrates a sweep of LFP signals (top trace) and the averaged LFP signals (bottom trace) induced by 8 -to- $12 \mathrm{~Hz}$ stimuli. The paired responses appear an increment pattern, i.e., response two is larger than response one. In statistical analysis, LFP amplitudes are significantly larger in response to $12 \mathrm{~Hz}$ stimuli than to $8 \mathrm{~Hz}$ ones $(p<0.05$; Figure 1D).

As an increasing frequency of stimulus-to-whisker makes neuronal responses enhanced, the network neurons discriminate the increases of input frequency through strengthening their responsiveness. How the neurons and
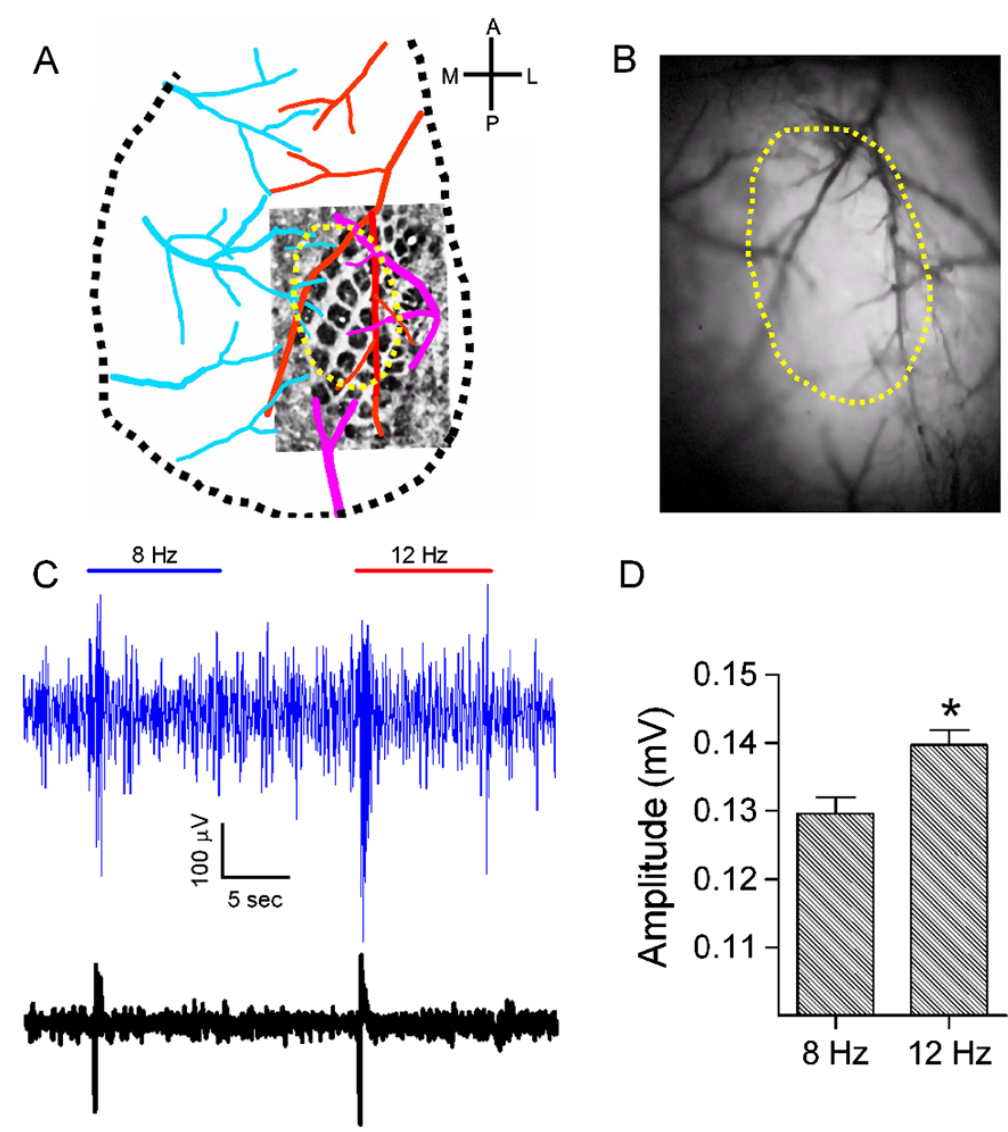

D

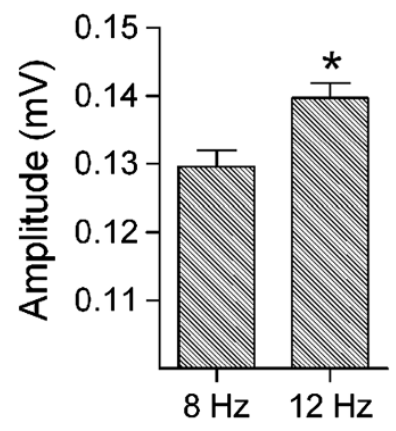

Figure 1 The activity of barrel neurons rises in response to an increased frequency of whisker tactile input. A) shows a spatial relationship between superfical vessels and barrel area in cerebral cortex. The veins are labeled in blue, the middle cerebral artery (mca) is labeled in red, and the others are pink. The location of barrel cortex is comfirmed by using histological reconstructions. B) shows an example of small craniotomy above the barrel cortex after removing a piece of skull. The cycles marked by yellow dash-line in A and B present the same area. C) shows an exmaple of LFPs induced by 8-to- $12 \mathrm{~Hz}$ stimuli (top) and the averaged trace of 20 LFP sweeps (bottom). D) shows LFP amplitiutdes at $12 \mathrm{~Hz}$ stimuli are significantly larger than those at $8 \mathrm{~Hz}$ stimili $(p<0.05$, students' test). 
astrocytes in the barrel cortex coordinately respond to the frequency messages was analyzed by two-photon calcium imaging in vivo.

\section{Neurons and astrocytes in barrel networks are} coordinately activated by whisker tactile inputs

After OGB-1 AM was loaded into neurons and astrocytes in the barrel cortex (Methods), cellular $\mathrm{Ca}^{2+}$ signals were measured by two-photon laser scanning microscopy. Left, middle and right panels in Figure 2A illustrate OBG-labeled nerve cells (green), SR101-labeled astrocytes (red) and their merged imaging (yellow for the astrocytes and greens for the neurons), respectively. Figure $2 \mathrm{~B}$ shows the stimulusinduced changes of $\mathrm{Ca}^{2+}$ signals $(\Delta \mathrm{F} / \mathrm{F})$ averaged from the neurons (green trace) and astrocytes (red), i.e., both of them respond to whisker tactile inputs. Pairswise cross-correlations between neurons and astrocytes in their activities (Methods) were analyzed under the conditions of whisker stimuli ( $\mathrm{n}=8$ mice) and no stimuli $(\mathrm{n}=6)$. Correlation coefficients with vs. without whisker stimuli are $0.40 \pm 0.03$ and $0.25 \pm 0.07$, respectively $(p<0.01$ in Figure $2 \mathrm{C})$, indicating that neurons and astrocytes in the barrel cortex are coordinately activated by whisker stimuli.

We further analyzed how neurons and astrocytes in the barrel cortex coordinately respond to frequency messages in whisker tactile inputs by measuring their spatial and temporal activities. This analysis is based on the data that the astrocytes in the brain regulate neuronal excitability and synaptic functions [34-36].

\section{Barrel neurons and astrocytes increase their activity} strength in response to high frequency input

In the study of the coordinate responses of neurons and astrocytes to frequency messages in whisker tactile inputs, we compared the changes of their activity strength induced by pair burst-stimuli to whiskers in frequencies 8to- $12 \mathrm{~Hz}$ (each burst for $10 \mathrm{sec}$. and inter-burst intervals in $10 \mathrm{sec}$.). Response one (R1) was corresponding to $8 \mathrm{~Hz}$ stimulus, and response two (R2) was a response to $12 \mathrm{~Hz}$. Compared to control (8-to-8 Hz) in Additional file 1: Figure $\mathrm{S} 1 \sim$ Additional file 2: Figure S2, an increased stimulus frequency appears to enhance the responsive strength in the neurons and astrocytes.

Figure 3 illustrates the three activity patterns of neurons and astrocytes in the barrel cortex. An example in 3A shows that most of the neurons express the increment in response to 8-to-12 Hz stimuli. The portions of decrement (R1 > R2), increment $(R 1<R 2)$ and parallel $(R 1=R 2)$ from all of the experiments $(n=6)$ are $21.5 \pm 9.9 \%$ (blue bar in $3 B$ ), $61.9 \pm 14.1 \%$ (red) and $16.6 \pm 6.5 \%$ (green), i.e., the portion of increment pattern is higher than others $(p<0.001)$. In addition, the responses of the astrocytes to 8-to- $12 \mathrm{~Hz}$
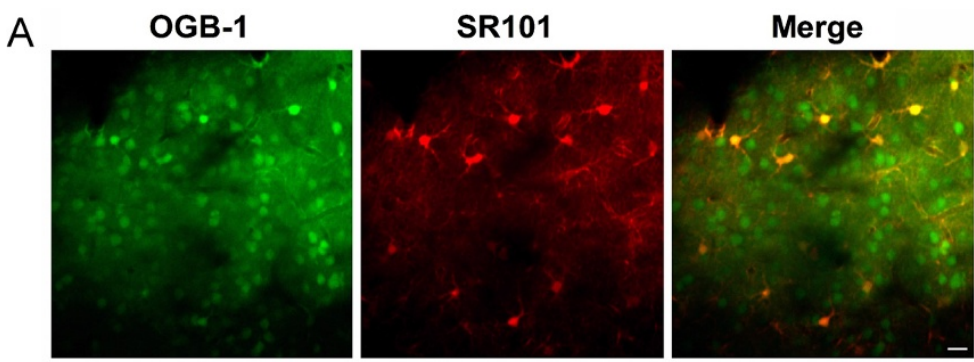

B
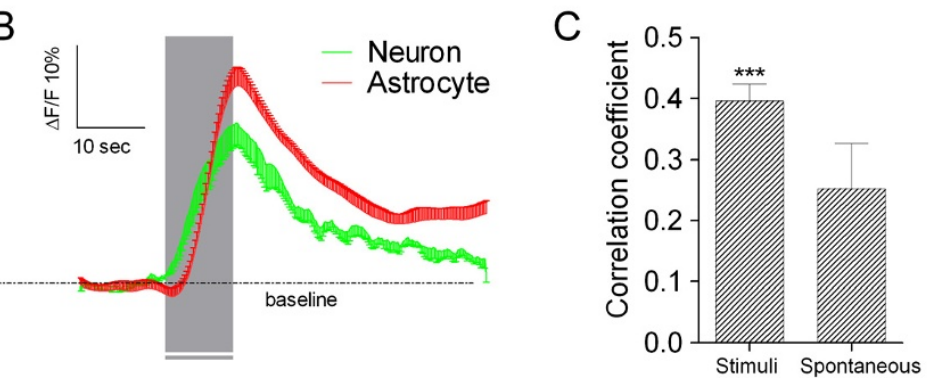

Figure 2 Whisker tactile inputs induce intracellular $\mathrm{Ca}^{2+}$ changes in the neurons and astrocytes of mouse barre cortex. A) shows twophoton images of these nerve cells (200 $\mathrm{\mu m}$ in the depth of barrel cortex), in which OGB-1 labels nerve cells (left panel), SR101 labels astrocytes (middle), and astrocytes are yellow in a merged image (right). Scale bar is $20 \mu \mathrm{m}$. B) shows the relative changes of OGB-1 fluorescence signals $(\Delta \mathrm{F} / \mathrm{F})$ averaged from the cell bodies of neurons (green line) and astroctyes (red) in a barrel network (gray bars are the durations of whisker stimuli). C) illustrates a comparison of neuron-astrocyte pairswise synchrony under the conditions with $(n=8)$ and without stimuli $(n=6)$. The synchrony of stimulus-driven activities is significantly higher than that of spontaneous ones $(p<0.01)$. The synchrony of cellular activities is quantified by using the peak cross-correlation coefficient. 

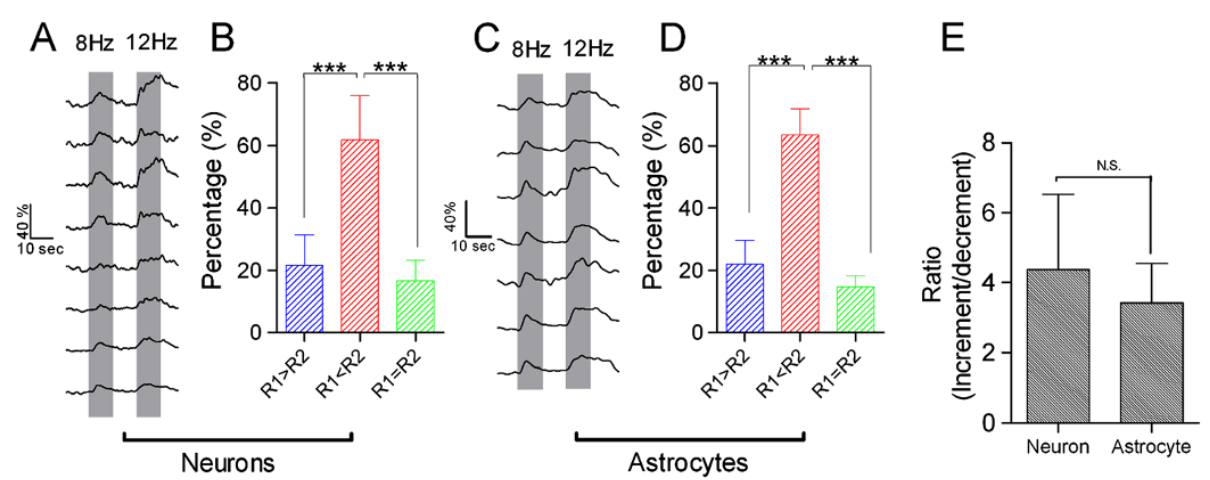

Figure 3 Barrel network neurons and astrocytes show a dominant increment in responses to the frequency increase of paired whisker stimuli. A) shows two sequential $\mathrm{Ca}^{2+}$ signals at the barrel neurons (a trace per cell) in response to paired burst-stimili at 8-to- $12 \mathrm{~Hz}$. B) The percentages of the three patterns among barrel network neurons ( $\mathrm{n}=5$ mice) in response to 8-to-12 $\mathrm{Hz}$ stimuli show the increment in dominance. C) $\mathrm{Ca}^{2+}$ signals in astrocytes are evoked by the paired burst-stimuli (gray bars) in 8-to-12 Hz. D) The increment in pair-responses (R1 versus R2) from astrocytes is dominant when the paired stimuli in 8-to- $12 \mathrm{~Hz}$ are given. E) The ratio of increment pattern to decrement patterns shows no significant difference between neuronal network and astrocytic network. Three asterisks indicate $p<0.001$, N.S. means no significant difference, student's t-test.

stimuli appear to be increment, decrement and parallel (Figure 3C). The portions in decrement, increment and parallel are $22.0 \pm 7.6 \%$ (blue bar in Figure 3D), $63.4 \pm 8.4 \%$ (red) and $14.6 \pm 3.7 \%$ (green), respectively $(p<0.001, \mathrm{n}=6$ ). Thus, a dominance of increment activity pattern of neurons and astrocytes is associated with an increasing frequency of whisker input.

We have calculated the ratio of increment portion to decrement one to estimate whether the responses of neurons and astrocytes to frequency-increased stimuli are parallel. As showed in Figure 3E, these ratios for neurons and astrocytes are not difference $(p=0.558)$. A parallel increase in the response strength of neurons and astrocytes indicates that the neurons and astrocytes in the barrel cortex coordinately respond to frequency messages in whisker tactile inputs.

\section{Barrel neurons and astrocytes synchronize their activities in response to high frequency input}

To study whether the activity synchrony of barrel neurons and astrocytes was related to identify frequency message in whisker inputs, we analyzed pair-wise correlation among cellpairs (Methods) in response to distinct frequencies. Each pixel in the matrices of Figure $4 \mathrm{~A} \sim \mathrm{B}$ and $4 \mathrm{D} \sim \mathrm{E}$ represents peak cross-correlation for a pair of neurons, and dark-red pixels indicate the best cross-correlation (synchrony), or vice versa.

An example in Figure $4 \mathrm{~A} \sim \mathrm{B}$ shows cross-correlations among neuron-pairs in response to burst-pulse one $(8 \mathrm{~Hz})$ and two $(12 \mathrm{~Hz})$. Correlation coefficients in central peaks averaged from all of the experiments $(\mathrm{n}=5)$ increase from $0.75 \pm 0.04$ for $8 \mathrm{~Hz}$ stimuli to $0.80 \pm 0.03$ for $12 \mathrm{~Hz}(p<0.05$, Figure $4 \mathrm{C})$. Neuronal activity synchrony corresponding to high frequency stimuli indicates that barrel neurons discriminate input frequency changes by setting their synchrony.
Figure $4 \mathrm{D} \sim \mathrm{E}$ shows an example that correlation coefficients among astrocyte-pairs increase in response to $8 \mathrm{~Hz}$-to-12 Hz. The peak values of the correlogram for $8 \mathrm{~Hz}$ stimuli vs. $12 \mathrm{~Hz}$ ones are $0.92 \pm 0.04$ and $0.96 \pm 0.03$, respectively $(p<0.05$, Figure $4 \mathrm{~F})$. The activity synchrony among the astrocytes in response to high frequency stimuli indicates that the barrel astrocytes modify their activity synchrony to encode the frequency message in whisker tactile inputs.

It is noteworthy that cells' responses in barrel cortex to pair-stimuli in the same frequency $(8-$ to- $8 \mathrm{~Hz}$ ) demonstrate decrement and less synchrony (Figures S1 $\sim$ S2). The result does not support a possibility that the increment and synchrony in their responses to stimulus two at $12 \mathrm{~Hz}$ are due to repetitive stimulation. Moreover, the parameters used to quantify active strength and synchrony are not affected by the interval of two stimuli in barrel neurons (Additional file 3: Figure S3).

\section{The neurons and astrocytes coordinately encode the frequency message in whisker tactile inputs}

Barrel neurons and astrocytes are synchronously activated by whisker tactile inputs (Figure 2C). The spatial and temporal patterns of barrel neurons and astrocytes are similar in response to frequency signals in whisker stimulations (Figures 3 4). We further examined whether the neurons and astrocytes in the barrel cortex coordinately encoded the frequency signals.

The cross-correlation for the pairs between neurons and astrocytes in response to whisker stimuli in 8-to$12 \mathrm{~Hz}$ was analyzed. Each pixel in Figure $5 \mathrm{~A} \sim \mathrm{B}$ represents peak cross-correlation for a pair of neuronastrocytes, and the cross-correlations appear to the increases in response to burst-stimuli one $(8 \mathrm{~Hz})$ and 

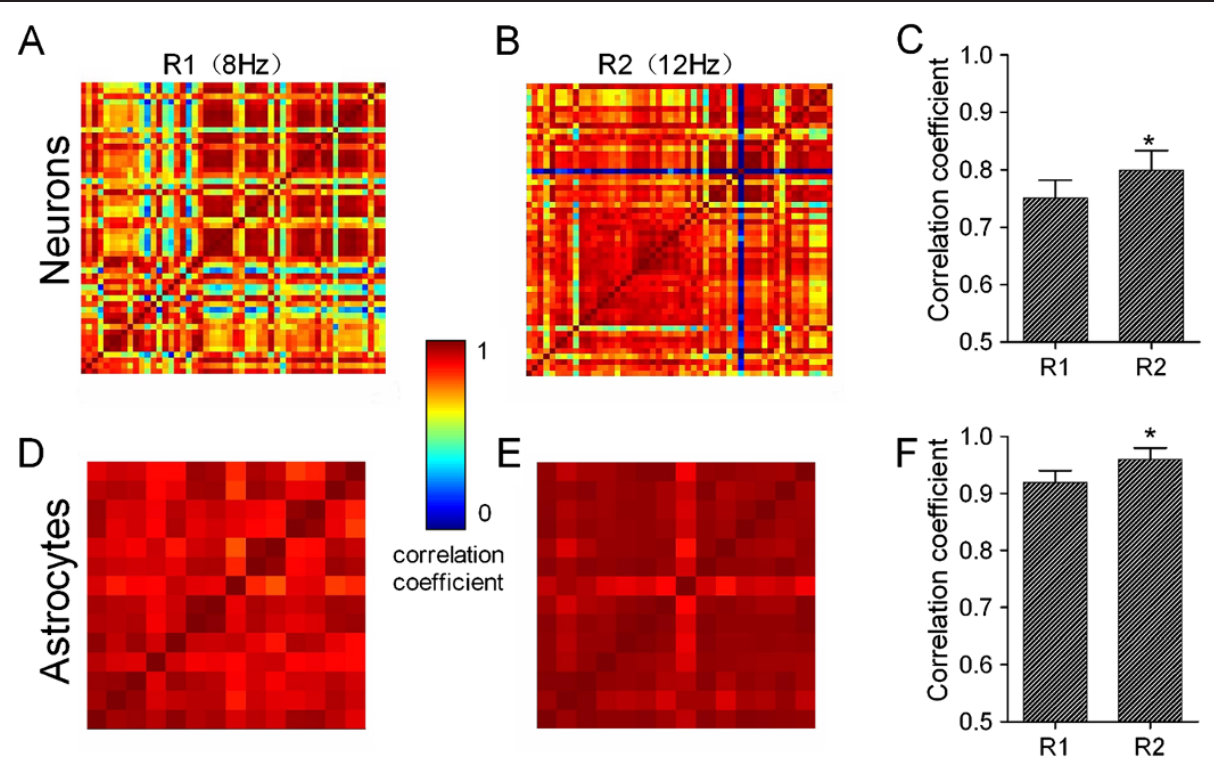

Figure 4 The activity synchrony of neuronal and astrocytic networks increases in responses to whisker stimuli with high frequency. A B) Correlation matrices show cross-correlations for each of neuron-pairs in response $\left(\mathrm{Ca}^{2+}\right.$ signals) to paired burst-stimuli, including response one (R1) vs. response two (R2) to $8 \mathrm{~Hz}(\mathrm{~A})$ and $12 \mathrm{~Hz}$ (B) of stimulus frequency, respectively. C) shows a comparison of correlation coefficients averaged from all active neuron-pairs (excluding auto-correlations) in responses to paired burst-stimuli at 8-to-12 Hz. D E) Correlation matrices show the cross-correlations for each of astrocyte-pairs in response to paired-stimuli, including R1 vs. R2 to $8 \mathrm{~Hz}$ (A) and $12 \mathrm{~Hz}$ (B) of stimulus frequencies, respectively. F) shows a comparison of the correlation coefficients averaged from all active astrocyte-pairs in responses to paired burst-stimuli (8-to-12 Hz). Asterisk presents $p<0.05$ in paired student's t-test.

two $(12 \mathrm{~Hz})$. Correlation coefficients in central peaks averaged from all of the experiments $(n=6)$ increase from $0.31 \pm 0.03$ for $8 \mathrm{~Hz}$ stimuli to $0.37 \pm 0.04$ for $12 \mathrm{~Hz}(p<0.05$, Figure $5 \mathrm{C})$. The more synchrony between neurons and astrocytes in response to the higher frequency stimuli indicates that neurons and astrocytes in the barrel cortices coordinately encode frequency messages in whisker tactile inputs.
The change of functional connections among nerve cells is involved in encoding input frequencies

Cellular mechanisms for barrel neurons and astrocytes coordinately to encode frequency messages in whisker tactile inputs is likely founded on the dynamical changes in their functional connections. If it is a case, the functional connections among nerve cells should be upregulated in response to an increase of stimulus frequency.
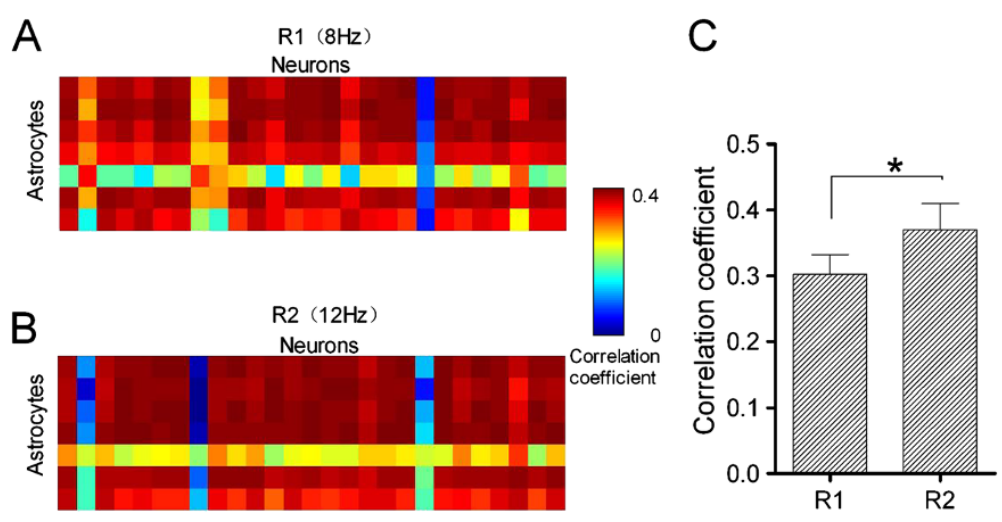

Figure $\mathbf{5}$ The activity synchrony of barrel neuron-astrocyte pairs is associated with a frequency increase of whisker stimuli. A B) Correlation matrices show the cross-correlations for each of neuron-astrocyte pairs in response $\left(\mathrm{Ca}^{2+}\right.$ signals) to paired-stimuli, including response one (R1) vs. response two (R2) at $8 \mathrm{~Hz}(\mathrm{~A})$ and $12 \mathrm{~Hz}$ (B) of stimulus frequencies, respectively. Vertical axis in correlation matrix shows for astrocytes and horizonal axis for neurons. $\mathrm{C}$ ) shows a comparison of correlation coefficients averaged from all active neuron-astrocyte pairs in responses to paired burst-stimuli at 8-to- $12 \mathrm{~Hz}$. Asterisk presents $p<0.05$ in paired student's t-test. 
The functional connections among these cells were estimated by using their cross-correlations [37,38]. Correlation coefficients during spontaneous activities at these nerve cells are set as no functional connections among them [39], and the averaged values above such coefficients plus two-time standard deviations are defined as the functional connections (Methods for details). Based on this principle, we analyzed the functional connections among neurons and astrocytes in the barrel cortex under the conditions of different input frequencies.

Figure 6 illustrates the functional connections among neurons in response to stimuli at $8 \mathrm{~Hz}$-to- $12 \mathrm{~Hz}$, in which $\mathrm{R} 1$ corresponds to $8 \mathrm{~Hz}$ and $\mathrm{R} 2$ to $12 \mathrm{~Hz}$. Figure $6 \mathrm{~A} \sim \mathrm{B}$ illustrates the functional connections (blue lines) among the neurons (green symbols) during R1 (A) and R2 (B). Figure $6 \mathrm{C}$ shows that the portions of the functionally connected neurons, in which $92 \pm 2.1 \%$ of the activated neurons are connected with others in $\mathrm{R} 1$, compared to $100 \%$ in $\mathrm{R} 2(p<0.05)$. Thus, more neurons in the barrel cortex are functionally connected in response to input frequency increase. Figure 6D shows the percentages of functional connections for each neuron with others in R1 and R2, which are $41.2 \pm 17.3 \%$ and $67.9 \pm 17.6 \%$, respectively ( $p$ $<0.05)$. That is, each of neurons is functionally connected with more neurons in response to an increase of input frequency. In short, the neurons in the barrel cortex encode the messages of the increased input frequencies by strengthening their functional connections.

Moreover, functional connections between neurons (green symbols) and astrocytes (reds) in response to stimuli at $8 \mathrm{~Hz}$ (Figure 7A) and $12 \mathrm{~Hz}$ (Figure 7B) were analyzed. The percentages of functional connections between neurons and astrocytes in $\mathrm{R} 1$ and $\mathrm{R} 2$ are $37.0 \pm 7.6 \%$ and $52 \pm 6.7 \%$, respectively (Figure $7 C, p<0.05$ ). Thus, the neurons and astrocytes in the barrel cortex coordinately encode an increase of input frequency via strengthening their functional connections.

\section{Discussion}

With two-photon cellular imaging and LFP recording in the barrel cortex in vivo, we have studied the processes for network cells to encode frequency messages in whisker
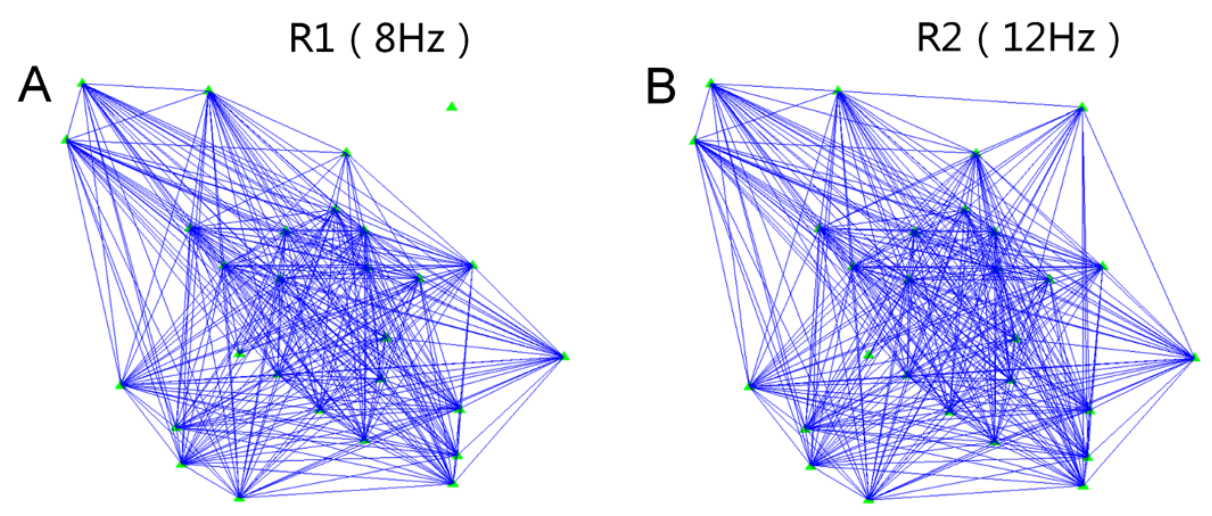

C

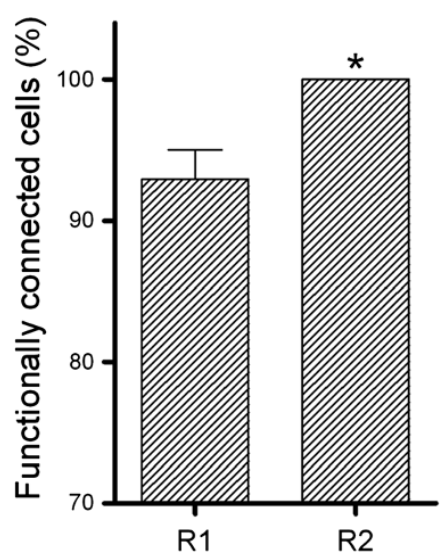

$\mathrm{D}$

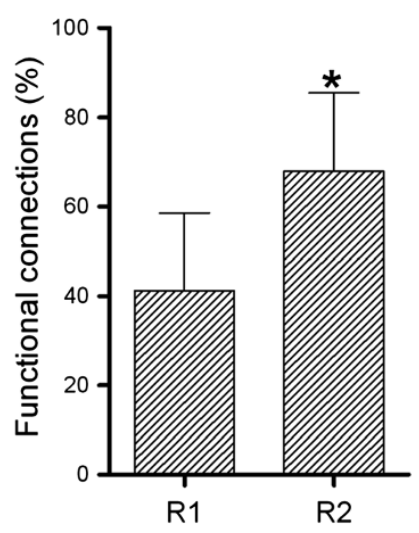

Figure 6 Functional connections among barrel neurons increase in response to stimulus frequencies at $8 \mathrm{~Hz}$ and $12 \mathrm{~Hz}$, in which response one (R1) corresponds to $8 \mathrm{~Hz}$ and response two (R2) corresponds to $12 \mathrm{~Hz}$. A B) presents the functional connections (blue lines) among the barrel neurons (green symbols) under the conditions of stimulus frequencies at $8 \mathrm{~Hz}(\mathbf{A})$ and $12 \mathrm{~Hz}$ (B), respectively. Scale bar is $20 \mu \mathrm{m}$. C) shows the percentages of functionally connected neurons in barrel networks during R1 (8 Hz input) and R2 (12 Hz). D) illustrates the percentage of functional connections for each neuron with others in R1 (8 Hz input) and R2 (12 Hz). Asterisk presents $p<0.05$ in paied student's t-test. 


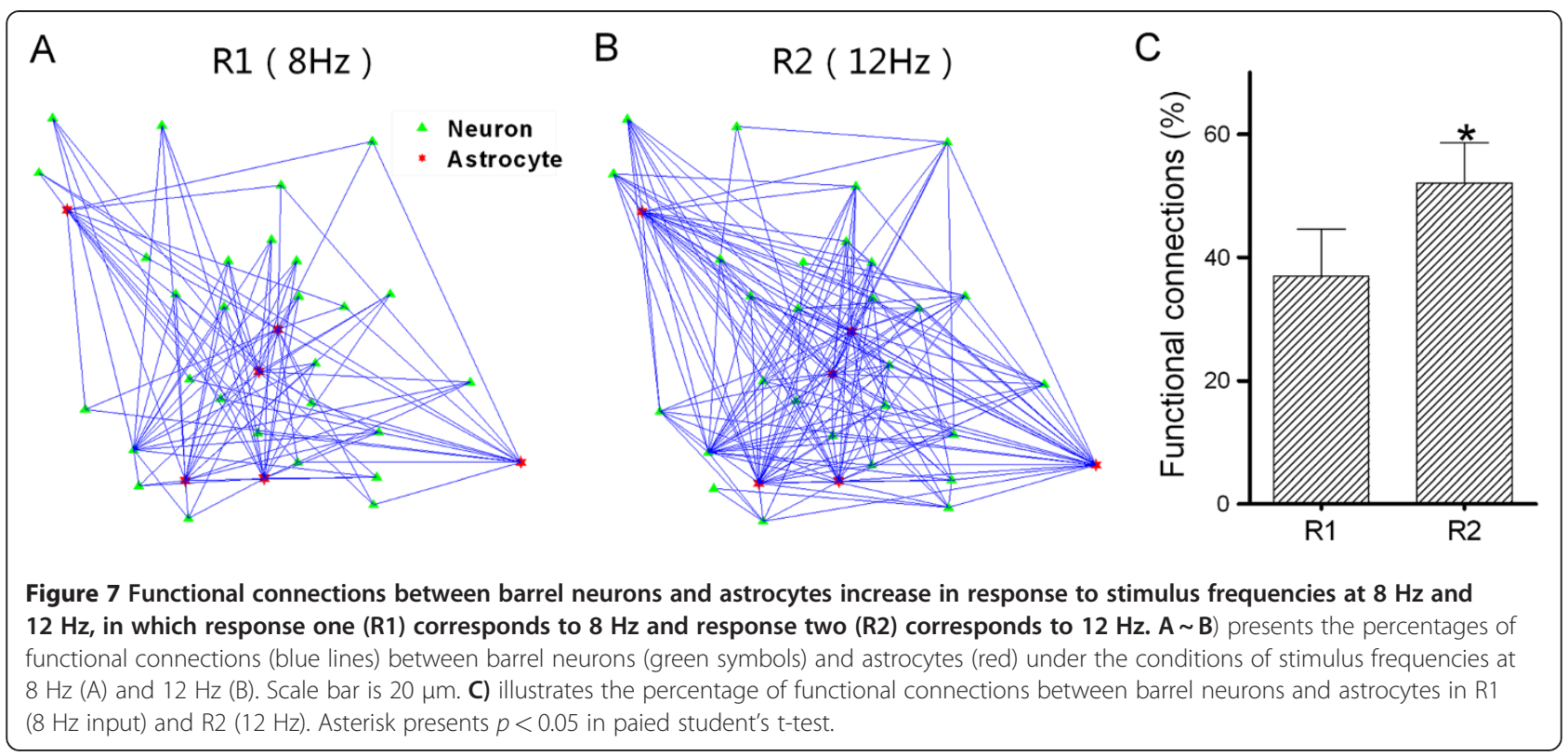

tactile inputs. In response to an increase of input frequency, the portions of neurons and astrocytes with increment activity patterns are dominant and the activities of neurons and astrocytes are synchronized (Figures 1 4), as well as the coordinate interactions between neuronal network and astrocytic one are strengthened (Figure 5). The process for these cells to encode an increase of input frequency is likely fulfilled by increasing their functional connections (Figures $6 \sim 7$ ). Thus, the neurons and astrocytes in the barrel cortex coordinately respond to the frequency messages in whisker tactile inputs through changing the strength of their functional connections. Whether this process is present in other sensory cortices remains to be examined.

In terms of the neural encoding, many studies have been done at individual neurons and synapses. Digital spikes encoded at the neurons and analogue signals at the synapses are essential codes in the brain [40-44]. As the brain functions are presumably fulfilled by neuronal networks [1-3], the studies in the activity patterns of network neurons have been paid attention [13,16,18,45]. Their temporal and spatial activity patterns in response in a network to a single stimulus are known [14,1719,21]. By giving stimuli in different frequencies, we reveal that nerve cells in the barrel cortex encode the frequency messages in whisker tactile inputs by resetting their functional connections as well as the portions of activity patterns and synchrony. Our findings provide the clues for understanding how neurons and astrocytes in the network coordinately program the brain codes for well-organized behaviors.

In the sensory systems, sequential stimuli in the same feature induce an adaptation in the sensations, and the increases in intensity or frequency should be given to elevate the sensitivity to these stimuli [46-48]. The changes in the ability of firing digital spikes at individual neurons are associated with the adaptation and sensitization $[23,49,50]$. It is not clear how the neurons and astrocytes integrated into neural networks encode these processes. Our studies demonstrate that pair-stimuli in the same frequency to whiskers make neurons and astrocytes to be lowered and asynchrony in their activities (Figures $\mathrm{S} 1 \sim \mathrm{S} 2$ ), and that an increasing stimulus frequency causes their responses to be increased and synchronized (Figures 2 4). The increment versus decrement and synchrony vs. asynchrony of nerve cells in response to sequential stimulations may be basic forms for encoding frequency signals and for the adaptation vs. sensitization. Their conversions may be involved in reading out the detail messages in natural sensory inputs.

The astrocytes presumably provide a micro-environment for the neurons to be functional $[10,35,36,51]$. Studies by two-photon cellular imaging show that sensory stimuli induce a change of $\mathrm{Ca}^{2+}$ signal in astrocytes [8] and that $\mathrm{Ca}^{2+}$ dynamics in the neurons of barrel cortex are synchrony $[4,7]$. The astrocytes play a crucial role in coupling neuronal organization to map signals in visual cortex [6]. Beyond these findings, we show that spatial and temporal activities among astrocytes are coupling with neuronal activities in strength and synchrony to encode frequency messages in whisker tactile inputs, which is based on the dynamical change of their functional connections.

It is noteworthy that the responses of barrel neurons and astrocytes may be thought to be different in the anesthetized animals versus awaked ones [52,53]. In our hypothesis, all responses of brain cells to stimulus intensity and frequency should be reduced in parallel from the anesthetized animals, compared to the awaked 
animals, such that the patterns of responses (i.e., response one versus response two) may not be changed. This hypothesis will be examined in our future studies.

\section{Conclusions}

Corresponding to an increase of whisker stimulus frequency, the neurons and astrocytes in the barrel cortex upregulate their activity strength, synchronize their activities and strengthen their functional connections. Therefore, the coordinate interactions among neurons and astrocytes in cortical networks are mechanistically involved in encoding the frequency messages in sensory inputs.

\section{Methods}

\section{Animal surgery and fluorescence labeling}

The study and all experiments conducted were fully approved by the Institutional Animal Care Unit Committee (IACUC) in Administration Office of Laboratory Animals Beijing China (ID\# B10831). FVB mice in postnatal days (PND) $20 \sim 35$ were anesthetized by the intraperitoneal injections of urethane $(1.5 \mathrm{~g} / \mathrm{kg})$. Anesthetic depth was judged based on lack of reflexes in pinch withdrawal and blink eyelid, and was maintained by giving the supplemental dosage of urethane $(0.25 \mathrm{~g} / \mathrm{kg})$ throughout the experiments. Body temperature was maintained by using a computer-controlled heating blanket at $37^{\circ} \mathrm{C}$. The barrel cortex was located based on the distributional map of superficial vessels and confirmed by histological reconstructions [54](Figure 1A). A craniotomy $(1 \sim 2 \mathrm{~mm}$ in diameter) was made on the skull above the center of the barrel cortex (Figure 1B), which was located at $1 \mathrm{~mm}$ posterior to the bregma and $3.5 \mathrm{~mm}$ lateral to the midline. It is noteworthy that the dura was intact throughout all experiments, and the care was taken to avoid any damage to superficial vessels and cortices.

In our studies, Oregon Green BAPTA-1-AM (OBG-1, Invitrogen, $\mathrm{CA}$ USA), a $\mathrm{Ca}^{2+}$ dye, was used to measure the activities from neurons and astrocytes. OGB-1 was dissolved in DMSO and 20\% Pluronic F-127 (2 g Pluronic F127 in $10 \mathrm{ml}$ DMSO, Invitrogen, USA) to have its stock solution at $10 \mathrm{mM}$. The stock solution was diluted in ACSF to yield its final concentration at $1 \mathrm{mM}$. This OBG-1 solution was injected into layer I $\sim$ II of barrel cortex by the pressure (1 bar, $5 \mathrm{~min})$ through a patch pipette $(200 \mu \mathrm{m}$ below the pia) to label the population of nerve cells, termed as multicell bolus loading [55-57]. In the meantime, $100 \mu \mathrm{M}$ sulforhodanmine-101 (SR101, Invitrogen, USA) was co-injected to label the astrocytes specifically [33]. The volumes of these dyes were controlled at $\sim 0.5 \mu \mathrm{l}$. After micro-injections, the craniotomy well was filled by lowmelted agarose $(1 \sim 2 \%)$ in saline and then was sealed with glass coverslip. The exposed skull was adhered to a custommade recording chamber with dental acrylic cement, which was surperfused with saline (mM): $125 \mathrm{NaCl}, 2.5 \mathrm{KCl}, 26$
$\mathrm{NaHCO}_{3}, 1.25 \mathrm{NaH}_{2} \mathrm{PO}_{4}, 2 \mathrm{CaCl}_{2}, 1 \mathrm{MgCl}_{2}, 20$ glucose (pH 7.4). The saline was warmed up to $37^{\circ} \mathrm{C}$ and bubbled with $95 \% \mathrm{O}_{2} / 5 \% \mathrm{CO}_{2}$.

\section{Two-photon cellular imaging}

Calcium imaging experiments were done 1 hour after dye injection by using two-photon laser scanning confocal microscope (Olympus FV-1000, Tokyo, Japan). Two-photon laser-beam generator (Mai Tai, Physical Spectrum, USA) and scanning system were mounted onto an upright microscope (Olympus BX61WI) with water immersion objectives (IR-LUMPLan Fl, 0.8NA, 40X). Two-photon laser beam at $810 \mathrm{~nm}$ was given to excite OGB-1 and SR101. Average power delivered to the brain tissue was less than $75 \mathrm{~mW}$. The emission wavelengths were $523 \mathrm{~nm}$ for $\mathrm{Ca}^{2+}$-binding OGB-1 and $603 \mathrm{~nm}$ for SR-101, respectively. Whole field images were acquired at $10 \mathrm{~Hz}$ frame rate $(256 \times 256$ pixels $)$. The parameters for laser beam and photomultiplier tube were locked for the measurements before and after different stimuli as well as throughout all experiments in order to have a consistent condition in the comparisons of the results.

\section{Local field potential (LFP) recording}

Local field potentials were recorded in layerII/III of barrel cortex by using a glass pipette that contained the standard pipette solution $(150 \mathrm{mM} \mathrm{NaCl}, 2.5 \mathrm{mM} \mathrm{KCl}, 10 \mathrm{mM}$ HEPES). The resistance of recording pipettes was $\sim 10 \mathrm{M} \Omega$. Electrical signals were inputted to an AxoClamp-2B amplifier and pClamp 10 system (Axon Instrument Inc. CA USA), in which Clampex was used for data acquisition and Clampfit for data analysis. The electrical signals were digitized at $10 \mathrm{kHz}$ and band-pass filtered at $1 \sim 100 \mathrm{~Hz}$. It is noteworthy that the LFP recording and two-photon cellular imaging were performed in the identical area of barrel cortex (Fig. $1 \mathrm{~A} \sim \mathrm{B}$ ), which allowed us to study the processes of neural encoding in the barrel cortex by electrophysiology and cellular imaging.

\section{Whisker stimuli and barrel cells' responses}

All major whiskers in the contralateral sides of the imaged barrel cortices were deflected in a caudal-to-rostral direction by air-puffing during the experiments, which is more similar to the natural movement of whiskers. Whisker stimuli were done by giving the sequential brief pulses of airpuffing ( $50 \mathrm{psi}, 50 \mathrm{~ms}$ ) through a tiny steel tube that was mounted on a micromanipulator and controlled with costume-made LabVIEW program. The stimulus patterns were the paired burst-pulses, in which each burst had a given frequency. The frequency patterns in these paired bursts (10 seconds for each) were 8 and $12 \mathrm{~Hz}$ (8-to- $12 \mathrm{~Hz}$ ), closely to natural frequency in exploratory whisking $[25,49,58]$. Burstpulse intervals were 10 seconds. To avoid the stimulations 
to the skin and furs, a tip of the stimulator was positioned in a way that it did not blow on the snout.

The amplitudes of $\mathrm{Ca}^{2+}$ signals are correlated positively with spike frequency, and $\mathrm{Ca}^{2+}$ levels in a neuron indicate its functional activities, so that spiking activity at the neurons can be estimated from their somatic $\mathrm{Ca}^{2+}$ signals in vivo $[19,31,32,59]$. Different from the spikes as a functional index of neuronal activity, the activities of astrocytes are associated with the changes of $\mathrm{Ca}^{2+}$ signals $[30,60]$. In addition, the synchrony of $\mathrm{Ca}^{2+}$ signals provide a measurement for the timing of cellular activities $[4,7,54,61]$. Therefore, we studied the activity patterns of barrel neurons and astrocytes in response to whisker stimuli by using in vivo two-photon cellular imaging in order to reveal the processes of neuronal encoding in brain networks, in which the peak amplitudes and temporal synchrony of $\mathrm{Ca}^{2+}$ signals were analyzed.

\section{Data analyses}

$\mathrm{Ca}^{2+}$ fluorescence signals for cellular responses to whisker stimuli were acquired by using Fluoviewer-10 software (Olympus Inc. Japan) and analyzed in the regions of interest (ROI) from cell bodies by using NIH ImageJ and MATLAB (MathWorks). To reduce the photon and PMT noise, a median filter (radius, 1 pixel) was applied to all images. $\mathrm{Ca}^{2+}$ signals in cellular responses were normalized and presented as relative fluorescence changes $(\Delta \mathrm{F} / \mathrm{F})[29,62,63]$. Baseline fluorescence (F) was an averaged value in the ROI before stimuli, and $\Delta \mathrm{F}$ values were differences between $\mathrm{Ca}^{2+}$ signals from the evoked responses in the ROI and baseline fluorescence. It is noteworthy that all of the fluorescence signals were subtracted from the noise signals of unstained blood vessels, as well as $\mathrm{Ca}^{2+}$ signals in the astrocytes were normalized to SR101 signal to eliminate motion artefacts. The normalized $\mathrm{Ca}^{2+}$ signals were smoothed by low-pass Butterworth filter to remove low-amplitude fluctuations and to minimize the distortions from fast $\mathrm{Ca}^{2+}$ transients [31,32]. Effective signals from each of active cells were judged according to a criterion that their relative fluorescence changes were greater than 2.5 SD of baseline values and lasted for $500 \mathrm{~ms}$. In our experiments, whisker stimuli induced the robust changes of $\mathrm{Ca}^{2+}$ signals in barrel cells, and the criteria above was found effective for sorting evoked signals from noise.

In our study, the paired burst-stimuli were given to induce two sequential responses. As the fluctuation of fluorescence signals influenced a precise measurement of response amplitudes, we thought the magnitude differences of two responses if their differences were above $2 \mathrm{SD}$ of baseline values; however, we defined no difference in the magnitude of two responses if their net changes were less than $2 \mathrm{SD}$. If cellular response one (R1) was larger than response two (R2) above $2 \mathrm{SD}$ of baseline, the pattern was defined as the decrement $(R 1>R 2)$. On the other hand, R2 $>$ R1 above 2
SD was the increment. No differences for R1 and R2 were called as parallel. This classification is similar to synaptic transmission patterns [43]. It is noteworthy that R2 values were the absolute changes of responses induced by stimulus two. That is, if the calcium signals in response to stimulus one were not back to their baseline levels, R2 was measured as a difference between the magnitude of response two and the residual level of response one.

The pairswise cross-correlations of normalized and smoothed $\mathrm{Ca}^{2+}$ signals $(\Delta \mathrm{F} / \mathrm{F})$ in the neurons and/or astrocytes between each of cell pairs were analyzed as Pearson correlation $[7,54,61,64]$. Although the cross-correlations between neurons from raw fluorescence traces were higher than the deconvolved traces over 2 fold [61], we computed raw traces without temporal deconvolution in the neurons consistently with those in astrocytes which had no spikes firing $[65,66]$. Consider two signals $\mathrm{x}(\mathrm{t})$ and $\mathrm{y}(\mathrm{t})$ of a real variable $\mathrm{t}$; the cross-correlation $r$ at delay $d$ is defined as:

$$
r=\frac{\sum_{t}[(x(t)-m x) \times(y(t-d)-m y)]}{\sqrt{\sum_{t}(x(t)-m x)^{2}} \times \sqrt{\sum_{t}(y(t-d)-m y)^{2}}}
$$

$m x$ and $m y$ are the means of the corresponding series. Correlation coefficients normalized to the autocorrelation at zero lag were calculated. Based on these calculations, the correlation matrices were plotted using MATLAB 7.0.

In the study of functional connectivity $[38,67]$ among network cells, we converted correlation coefficient matrix $(r)$ into binary adjacency matrix $(A)$ by setting a threshold (thresh) [68,69]. It was the averaged correlation coefficients plus two-time standard deviations corresponding to spontaneous cellular activities without whisker stimuli. If $r_{i j}$ during whisker stimuli is lareger than thresh, i.e., $A_{i, j}$ equals to one, the functional connection is present between cell $i$ and cell $j$. On the other hand, if $r_{i j}$ during whisker stimuli is less than thresh, i.e., $A_{i j}$ is equal to zero, the functional connection is not present between cell $i$ and cell $j$. The formula is

$$
A_{i, j}=\left\{\begin{array}{c}
1, r_{i j}>\text { thresh } \\
0, \text { otherwise. }
\end{array}\right.
$$

It is noteworthy that the definition of functional connection, whose threshold is set at mean $+2 \mathrm{SD}$ of correlation coefficients during the spontaneous activities of network cells, is based on an assumption that their activities are random in nature (no coordination). In other words, there are no interactions, or functional connections, among these network cells without input signals [70].

Based on these criteria of binary adjacency matrices and the spatial positions of network cells, we plotted the graphs which consisted of a set of nodes (the cells activated by stimulus bursts) and their functional connections (lines) 
under the conditions of response one to $8 \mathrm{~Hz}$ stimuli and response two to $12 \mathrm{~Hz}$. In these graphs of neural networks, two parameters for each cell were merited to indicate how each cell is connected with others. The cell that connects with one at least is called as a function-connected cell in neural network. The percentages of function-connceted cells present how many cells are functionally connected with others. If a cell connects with others, the percentages of functional connections for its actually connected cells in the total cells are calculated to present the connection strength for each of network cells. The folmula are given below.

For a neural network consisting of activated neurons $(N)$ in complete graphs, the number of connctions for each neuron with others is $N-1$. The number of functionconnected neurons is $n$, and the averaged number of connections for each neuron with others is $k$. Thus, $P_{n}=n / N$ stands for the percentages of function-connected neurons. $P_{k}=k /(N-1)$ presents the percentages of functional connections of each neuron. The astrocytes are connected tightly and widely via gap junctions, the number of connected neuron-astrocyte pairwise shows no variation during different stimuli in our studies. In a network comprising of $N$ neurons and $M$ astrocytes, we calculate the percentages of neuron-astrocytic functional connections $(l)$ in total potential links, which is $P_{l}=l /(N \cdot M)$.

All data are presented as mean \pm SEM. Student's t tests (two-tailed, paired, or unpaired assuming unequal variances) were done in $\mathrm{R}$ software package, version 2.10.1 (http://www.r-project.org/) to evaluate statistical significance. A $p \leq 0.05$ is defined as statistical significance.

\section{Additional files}

Additional file 1: Figure S1. Barrel network neurons show a dominant decrement in responses to the same frequency of paired whisker stimuli. A) shows two sequential $\mathrm{Ca}^{2+}$ signals at barrel neurons (a trace per cell) in response to paired burst-stimili at 8 -to- $8 \mathrm{~Hz}$. B) The paired burst-stimuli induce increment ( $n=2 / 24$; blue symbols in top panel), decrement ( $n=18 / 24$; reds in middle) and parallel ( $n=4 / 24$; greens in bottom) \#\#\# shows $p<0.001$ in two-tail paired t-test.

Additional file 2: Figure S2. The activity synchrony of barrel neurons decreases in correspondent to whisker stimuli with the same frequency. A B) Correlation matrices show the correlation coefficients for each of neuron-pairs in response (i.e., $\mathrm{Ca}^{2+}$ signals) to paired-stimuli, including response one (R1) vs. response two (R2) to stimuli both at $8 \mathrm{~Hz}$. C) shows the comparison of mean cross-correlation coeffiecent for all active neuron-pairs (excluding autocorrelations) in responses R1 and R2 to paired burst-stimuli at 8-to-8 $\mathrm{Hz}$.

Additional file 3: Figure S3. The interval of the whisker stimuli does not affect the measurement of activity patterns and synchrony. A-B) shows the averaged responses in a barrel neuronal network induced by the 8-to- $12 \mathrm{~Hz}$ whisker stimuli with different intervals. The stimuli interval of the panel $\mathbf{A}$ is $10 \mathrm{sec}$ and $\mathbf{B}$ is $60 \mathrm{sec}$. C) Both the short interval (10sec) and long interval (60sec) of the 8 -to- $12 \mathrm{~Hz}$ stimuli induces a dominant decrement in barrel neurons ( $p<0.001$, student's test). $D$ ) shows the comparision of mean cross-correlation coeffiecent for all active neuron-pairs in responses R1, R2 (responses induced by the second stimuli after a short interval ) and R2' (responses induced by the second stimuli after a long interval ). The coeffiecent of R1 and R2 are significantly higher than the one of R1
( $P<0.05$, student's test). The coffeicents of R2 and R2' shows non significant difference.

\section{Competing interests}

The authors declare that they have no competing interests.

\section{Acknowledgement}

We thank Dr. Keegan's proof reading before the submission. This study is granted by National Award for Outstanding Young Scientist (30325021), Natural Science Foundation China $(30990261,81171033)$ and National Basic Research Program (2011CB504405) to JHW

\section{Author details}

'Institute of Biophysics, Chinese Academy of Sciences, Beijing, 100101 China. ${ }^{2}$ Graduate School of the Chinese Academy of Sciences, Beijing, 100049 China. ${ }^{3}$ College of Life Science, University of Science and Technology of China, Hefei, Anhui 230026 China.

\section{Authors' contributions}

JZ and JHW conceived the idea, designed experiments, and wrote the paper. JZ and DW executed experiments and analyzed data. All authors read and approved the manuscript.

Received: 21 March 2012 Accepted: 26 April 2012

Published: 26 April 2012

\section{References}

1. Byrne JH: Learning and memory: basic mechanisms. Amsterdam: Academic; 2003.

2. Kandel E, Schwartz J, Jessell T: Principles of Neural Science. 4th edition. New York: McGraw-Hill; 2000.

3. Shepard GM: Neurobiology. Oxford: Oxford University Press; 1994

4. Hirase H, Qian L, Bartho P, Buzsaki G: Calcium dynamics of cortical astrocytic networks in vivo. PLOS Biol 2004, 2:E96.

5. Gourine AV, Kasymov V, Marina N, Tang F, Figueiredo MF, Lane S, Teschemacher AG, Spyer KM, Deisseroth K, Kasparov S: Astrocytes control breathing through pH-dependent release of ATP. Science 2010, 329:571-575.

6. Schummers J, Yu H, Sur M: Tuned responses of astrocytes and their influence on hemodynamic signals in the visual cortex. Science 2008, 320:1638-1643.

7. Takata $\mathrm{N}$, Hirase $\mathrm{H}$ : Cortical layer 1 and layer $2 / 3$ astrocytes exhibit distinct calcium dynamics in vivo. PLOS One 2008, 3:e2525

8. Wang X, Lou N, Xu Q, Tian G-F, Peng WG, Han X, Kang J, Takano T, Nedergaard M: Astrocytic Ca2+ signaling evoked by sensory stimulation in vivo. Nat Neurosci 2006, 9:816-823.

9. Christopherson KS, Ullian EM, Stokes CC, Mullowney CE, Hell JW, Agah A, Lawler J, Mosher DF, Bornstein P, Barres BA: Thrombospondins are astrocyte-secreted proteins that promote CNS synaptogenesis. Cell 2005, 120:421-433.

10. Fellin T: Communication between neurons and astrocytes: relevance to the modulation of synaptic and network activity. J Neurochem 2009, 108:533-544.

11. Giaume C, Maravall M, Welker E, Bonvento G: The Barrel Cortex as a Model to Study Dynamic Neuroglial Interaction. Neuroscientist 2009 15:351-366.

12. Haber M, Zhou L, Murai KK: Cooperative astrocyte and dendritic spine dynamics at hippocampal excitatory synapses. J Neurosci 2006, 26:8881-8891.

13. Gobel W, Helmchen F: In vivo calcium imaging of neural network function. Physiology (Bethesda) 2007, 22:358-365.

14. Ohki K, Chung S, Ch'ng YH, Kara P, Reid RC: Functional imaging with cellular resolution reveals precise micro-architecture in visual cortex. Nature 2005, 433:597-603.

15. Rochefort NL, Garaschuk O, Milos RI, Narushima M, Marandi N, Pichler B, Kovalchuk Y, Konnerth A: Sparsification of neuronal activity in the visual cortex at eye-opening. Proc Natl Acad Sci U S A 2009.

16. Svoboda K, Yasuda R: Principles of two-photon excitation microscopy and its applications to neuroscience. Neuron 2006, 50:823-839. 
17. Kerr JN, de Kock CP, Greenberg DS, Bruno RM, Sakmann B, Helmchen F: Spatial organization of neuronal population responses in layer $2 / 3$ of rat barrel cortex. J Neurosci 2007, 27:13316-13328.

18. Kerr JN, Greenberg D, Helmchen F: Imaging input and output of neocortical networks in vivo. Proc Natl Acad Sci U S A 2005, 102:14063-14068.

19. Sato TR, Gray NW, Mainen ZF, Svoboda K: The Functional Microarchitecture of the Mouse Barrel Cortex. PLoS Biol 2007, 5:e189.

20. Stettler DD, Axel R: Representations of odor in the piriform cortex. Neuron 2009, 63:854-864.

21. Winship IR, Plaa N, Murphy TH: Rapid astrocyte calcium signals correlate with neuronal activity and onset of the hemodynamic response in vivo. J Neurosci 2007, 27:6268-6272.

22. Ahl AS: The role of vibrissae in behavior: a status review. Vet Res Commun 1986, 10:245-268.

23. Melzer P, Sachdev RN, Jenkinson N, Ebner FF: Stimulus frequency processing in awake rat barrel cortex. J Neurosci 2006, 26:12198-12205.

24. Melzer P, Champney GC, Maguire MJ, Ebner FF: Rate code and temporal code for frequency of whisker stimulation in rat primary and secondary somatic sensory cortex. Exp Brain Res 2006, 172:370-386.

25. Moore $\mathrm{Cl}$ : Frequency-dependent processing in the vibrissa sensory system. J Neurophysiol 2004, 91:2390-2399.

26. Petersen CC: The functional organization of the barrel cortex. Neuron 2007, 56:339-355.

27. Sato TR, Svoboda K: The functional properties of barrel cortex neurons projecting to the primary motor cortex. J Neurosci 2010, 30:4256-4260.

28. Svoboda K, Helmchen F, Denk W, Tank DW: Spread of dendritic excitation in layer $2 / 3$ pyramidal neurons in rat barrel cortex in vivo. Nat Neurosci $1999,2: 65-73$

29. Cossart R, lkegaya $Y$, Yuste R: Calcium imaging of cortical networks dynamics. Cell Calcium 2005, 37:451-457.

30. Di Garbo A, Barbi M, Chillemi S, Alloisio S, Nobile M: Calcium signalling in astrocytes and modulation of neural activity. Biosystems 2007, 89:74-83.

31. Moreaux $L$, Laurent $G$ : Estimating firing rates from calcium signals in locust projection neurons in vivo. Front Neural Circuits 2007, 1:2.

32. Yaksi $E$, Friedrich RW: Reconstruction of firing rate changes across neuronal populations by temporally deconvolved Ca2+ imaging. Nat Methods 2006, 3:377-383.

33. Nimmerjahn A, Kirchhoff F, Kerr JN, Helmchen F: Sulforhodamine 101 as a specific marker of astroglia in the neocortex in vivo. Nat Methods 2004, 1:31-37.

34. Lin SC, Bergles DE: Synaptic signaling between neurons and glia. Glia 2004, 47:290-298.

35. Schipke CG, Kettenmann H: Astrocyte responses to neuronal activity. Glia 2004, 47:226-232.

36. Vernadakis A: Glia-neuron intercommunications and synaptic plasticity. Prog Neurobiol 1996, 49:185-214.

37. Cho MW, Choi MY: Brain Networks: Graph Theoretical Analysis and Development Models. Int J Imag Syst Tech 2010, 20:108-116.

38. Foldy C, Dyhrfjeld-Johnsen J, Soltesz I: Structure of cortical microcircuit theory. J Physiol 2005, 562:47-54.

39. Ringach DL: Spontaneous and driven cortical activity: implications for computation. Curr Opin Neurobiol 2009, 19:439-444.

40. Abbott LF, Regehr WG: Synaptic computation. Nature 2004, 431:796-803.

41. Chen N, Chen X, Wang JH: Homeostasis established by coordination of subcellular compartment plasticity improves spike encoding. J Cell Sci 2008, 121:2961-2971.

42. Chen N, Yu J, Qian H, Ge R, Wang JH: Axons amplify somatic incomplete spikes into uniform amplitudes in mouse cortical pyramidal neurons. PLoS One 2010, 5:e11868

43. Wang JH, Wei J, Chen X, Yu J, Chen N, Shi J: Gain and fidelity of transmission patterns at cortical excitatory unitary synapses improve spike encoding. J Cell Sci 2008, 121:2951-2960.

44. Yu J, Qian H, Chen N, Wang JH: Quantal glutamate release is essential for reliable neuronal encodings in cerebral networks. PLoS One 2011, 6:e25219.

45. Brecht M, Fee MS, Garaschuk O, Helmchen F, Margrie TW, Svoboda K, Osten $P$ : Novel approaches to monitor and manipulate single neurons in vivo. J Neurosci 2004, 24:9223-9227.
46. Jones EG: Cortical and subcortical contributions to activity-dependent plasticity in primate somatosensory cortex. Annu Rev Neurosci 2000, 23:1-37

47. Karwoski CJ, Proenza LM: Transient Adaptation and Sensitization in the Retina of Necturus. J Gen Physiol 1980, 76:479-497.

48. Teller DY, Gestrin PJ: Sensitization by Annular Surrounds. Sensitization and Dark Adaptation. Vision Res 1969, 9:1481-1489.

49. Kleinfeld D, Ahissar E, Diamond ME: Active sensation: insights from the rodent vibrissa sensorimotor system. Curr Opin Neurobiol 2006, 16:435-444.

50. Maravall M, Petersen RS, Fairhall AL, Arabzadeh E, Diamond ME. Shifts in coding properties and maintenance of information transmission during adaptation in barrel cortex. PLOS Biol 2007, 5:e19.

51. Newman EA: New roles for astrocytes: regulation of synaptic transmission. Trends Neurosci 2003, 26:536-542.

52. Hartings JA, Simons DJ: Thalamic relay of afferent responses to 1- to 12-Hz whisker stimulation in the rat. J Neurophysiol 1998, 80:1016-1019.

53. Vijayan S, Hale GJ, Moore Cl, Brown EN, Wilson M: Activity in the barrel cortex during active behavior and sleep. J Neurophysiol 2010, 103:2074-2084

54. Golshani P, Goncalves JT, Khoshkhoo S, Mostany R, Smirnakis S, Portera-Cailliau C: Internally mediated developmental desynchronization of neocortical network activity. J Neurosci 2009, 29:10890-10899.

55. Garaschuk O, Milos RI, Konnerth A: Targeted bulk-loading of fluorescent indicators for two-photon brain imaging in vivo. Nat Protoc 2006 1:380-386.

56. Golshani $P$, Portera-Cailliau C: In vivo 2 -photon calcium imaging in layer $2 / 3$ of mice. J Vis Exp 2008, 13:681.

57. Stosiek C, Garaschuk O, Holthoff K, Konnerth A: In vivo two-photon calcium imaging of neuronal networks. Proc Natl Acad Sci U S A 2003 100:7319-7324.

58. Ni H, Huang L, Chen N, Zhang F, Liu D, Ge M, Guan S, Zhu Y, Wang JH: Upregulation of barrel GABAergic neurons is associated with cross-modal plasticity in olfactory deficit. PLoS One 2010, 5:e13736.

59. Petersen $\mathrm{OH}$, Michalak $\mathrm{M}$, Verkhratsky A: Calcium signalling: past, present and future. Cell Calcium 2005, 38:161-169.

60. Halassa MM, Fellin T, Haydon PG: The tripartite synapse: roles for gliotransmission in health and disease. Trends Mol Med 2007, 13:54-63.

61. Smith SL, Hausser M: Parallel processing of visual space by neighboring neurons in mouse visual cortex. Nat Neurosci 2010, 13: 114-1149.

62. Stutzmann GE, Parker I: Dynamic multiphoton imaging: a live view from cells to systems. Physiology 2005, 20:15-21.

63. Knopfel T, Diez-Garcia J, Akemann W: Optical probing of neuronal circuit dynamics: genetically encoded versus classical fluorescent sensors. Trends Neurosci 2006, 29:160-166.

64. Ch'ng YH, Reid RC: Cellular imaging of visual cortex reveals the spatial and functional organization of spontaneous activity. Front Integr Neurosci 2010, 420.

65. Deitmer JW, Verkhratsky AJ, Lohr C: Calcium signalling in glial cells. Cell Calcium 1998, 24:405-416.

66. Nedergaard M, Rodriguez JJ, Verkhratsky A: Glial calcium and diseases of the nervous system. Cell Calcium 2010, 47:140-149.

67. Eldawlatly $\mathrm{S}$, Jin $\mathrm{R}$, Oweiss KG: Identifying functional connectivity in large-scale neural ensemble recordings: a multiscale data mining approach. Neural Comput 2009, 21:450-477.

68. Sporns O: Small-world connectivity, motif composition, and complexity of fractal neuronal connections. Biosystems 2006, 85:55-64.

69. Zhou D, Thompson WK, Siegle G: MATLAB toolbox for functional connectivity. Neurolmage 2009, 47:1590-1607.

70. Sporns O: The non-random brain: efficiency, economy, and complex dynamics. Front Comput Neurosci 2011, 5:5.

doi:10.1186/1756-6606-5-12

Cite this article as: Zhao et al.: Barrel cortical neurons and astrocytes coordinately respond to an increased whisker stimulus frequency. Molecular Brain 2012 5:12. 